\title{
Precision In Situ Control of Local Liquid Chemistry via Electron Irradiation
}

\author{
C. Wang ${ }^{1}$, T. Shokuhfar ${ }^{1,2}$, and R.F. Klie ${ }^{1}$ \\ 1. Dept. of Physics, University of Illinois at Chicago, Chicago, IL, USA 60607 \\ 2. Dept. of Mechanical Engineering, Dept. of Biomedical Engineering, Michigan Technological \\ University, Houghton, MI, USA 49931
}

In situ liquid electron microscopy has allowed direct observations of biological [1], electrochemical [2], physical [3], or chemical processes [4] near or at atomic resolution. Previous studies have focus on reporting various sample behavior under different electron microscope conditions. In this report, we discuss the possibility of utilizing electron irradiation to precisely control the liquid chemical reactivity by controlling the electron induced radical concentration. Since space-time resolution of the real-time observation is limited by electron dose rate, for higher resolution a higher electron dose rate is always desired. This, however contradicts the need to suppress either particular, or all types of beam effects caused by electron-induced radical species under high absorbed dose rate. By taking advantage of the nonlinear effects in the electron water interaction, we were able to suppress the steady state concentration of electron beam induced species at high electron dose rate, allowing high resolution electron microscopy and spectroscopy while minimizing the damage to the sample. On the other hand, by changing the liquid cell design, we were able to suppress the formation of hydrogen gas bubble while maintaining a high free radical concentration, allowing applications taking advantage of these radicals while avoiding phase change of the liquid environment.

The electron beam induced radical species are often undesired as they can cause physical or chemical change to the sample. Figure 1 shows a dose rate series experiment conducted on graphene sandwiched ferritin molecules in STEM mode. We find that ferritin protein denatures into different types of subunits under different electron dose rate while maintaining an overall intact structure below a threshold of $6 \mathrm{e}^{-} / \AA^{2} / \mathrm{s}$. Previous studies have also shown that many other beam effects, such as formation of bubble [1] or the reduction of metal nanoparticles [4], can be prevented by maintaining the electron dose rate at a level below the reaction threshold, which, as suggested by electron water interaction simulation [5], can be explained by a low steady state concentration of electron generated radical species. However, in real time observations this low dose rate approach imposes a theoretical limit on the attainable spacetime resolution, which is inversely proportional to the square of the electron dose rate. We introduce an electron pulse imaging approach to suppress the generated radical concentration under a time averaged high dose rate. Combined with simulation and experiment, we find that by turning off the electron pulse before the generated radicals reach threshold concentration, as well as allowing enough time for the sample to relax before the subsequent pulse, it is possible to maintain a below-threshold radical concentration at a higher time-averaged electron dose rate, which improves the space-time resolution for real-time observations free of beam effects. Using electron induced hydrogen bubble formation as an indicator, we showed that by optimizing the probe current, pulse time and relaxation time combination, the time averaged threshold electron dose rate to reach the saturation condition for the dissolved hydrogen gas is significantly increased. Simulation confirmed an overall lower concentration of all radical species produced by this method, which allows atomic resolution imaging free of bubble formation and other beam effects, as we have qualitatively shown in our previous paper [1]. 
On the other hand, electron induced radical can be beneficial when an increased chemical activity in the liquid is desired. Previous experiments [1,4] and simulations [5] both suggested that a higher electron dose rate in electron microscopy is analogous to a higher concentration of reacting agent in conventional liquid chemistry, resulting a more reactive environment for applications such as in situ nanoparticle synthesis. Although the existence of the steady state provides a convenient way of precision control of liquid chemistry in terms of concentration of radical species via control of electron dose rate, the extend of this approach remain limited due to bubble formation under moderate dose rate. To take advantage of the increased beam induced chemical activity, bubble formation needs to be suppressed while maintaining a high overall radical concentration, which also means a high dissolved hydrogen gas concentration. We find that increasing the saturation threshold will significantly increases the bubble formation threshold dose rate, allowing an electron-induced chemically reactive liquid environment free of gas bubble condensation.

\section{References:}

[1] Wang, C., et al, Advanced Materials 26.21 (2014), 3410-3414.

[2] Sacci, R. L., et al, Chemical Communications 50.17 (2014), 2104-2107.

[3] Chen, Q., et al, Faraday discussions 175 (2014), 203-214.

[4] Woehl, T. J., et al, ACS nano 6.10 (2012), 8599-8610.

[5] Schneider, N. M., et al, The Journal of Physical Chemistry C 118.38 (2014) 22373-22382.

[6] This research was supported by Michigan Technological University and Research Resource Center, University of Illinois at Chicago.
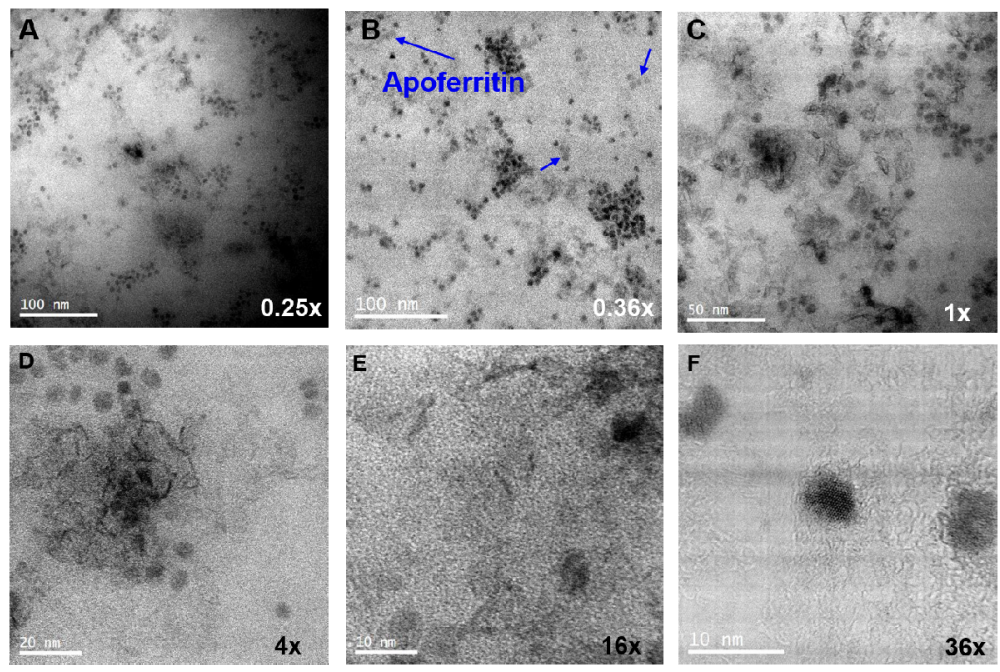

Figure 1: ABF images of sandwiched ferritin molecules at different area averaged dose rates. The area averaged dose rate used to acquire each image is gradually increased with the value of each image being (A): $1.4 \mathrm{e}^{-} / \AA^{2} / \mathrm{s}(\sim 0.25 \times) ;(\mathbf{B}): 2.2 \mathrm{e}^{-} / \AA^{2} / \mathrm{s}(\sim 0.36 \times)[1] ;(\mathbf{C}): 6 \mathrm{e}^{-} / \AA^{2} / \mathrm{s}(1 \times) ;(\mathbf{D}): 23 \mathrm{e}^{-} / \AA^{2} / \mathrm{s}(\sim 4 \times)$; (E): $91 \mathrm{e}^{-/} / \AA^{2} / \mathrm{s}(\sim 16 \times)$; (F): $143 \mathrm{e}^{-/} / \AA^{2} / \mathrm{s}(\sim 25 \times)$. The number on the lower right corner of each image is the dose rate multiple compared to the threshold dose rate in $(\mathbf{B})$. The denaturation process of ferritin protein became visible at a dose rate of $6 \mathrm{e}^{-/} \AA^{2} / \mathrm{s}$ as shown in image (B). The diameters of the sub-units in Figure 5B-E are measured to be between $0.4 \mathrm{~nm}$ to $1.6 \mathrm{~nm}$. We identified these structure as polypeptides, sub-units of the ferritin protein shell. As shown in image $(\mathbf{F})$, the protein is further broken down into biological structures with a width of approximately $1.3 \AA$, which we identified as amino acids at a dose rate of $206 \mathrm{e}^{-} / \AA^{2} / \mathrm{s}\left(\sim 36\right.$ times of the denaturation initiating dose rate $\left.6 \mathrm{e}^{-} / \AA^{2} / \mathrm{s}\right)$. 